\title{
Fluidized Bed Layer-by-Layer Microcapsule
}

\section{Formation}

Joseph J. Richardson, Darwin Teng, Mattias Björnmalm, Sylvia T. Gunawan, Junling Guo, Jiwei

Cui, George V. Franks, Frank Caruso*

Department of Chemical and Biomolecular Engineering, The University of Melbourne, Parkville, Victoria 3010, Australia

\begin{abstract}
Polymer microcapsules can be used as bioreactors and artificial cells; however, preparation methods for cell-like microcapsules are typically time consuming, low yielding and/or involve custom microfluidics. Here, we introduce a rapid ( 30 min per batch, eight layers), scalable (up to $500 \mathrm{mg}$ of templates) and efficient (98\% yield) microcapsule preparation technique utilizing fluidized bed for the layer-by-layer (LbL) assembly of polymers, and we investigate the parameters that govern the formation of robust capsules. Fluidization in water was possible for particles of comparable diameter to mammalian cells $(>5 \mu \mathrm{m})$, with the experimental flow rates necessary for fluidization matching well with the theoretical values. Important variables for polymer film deposition and capsule formation were the concentration of polymer solution and the molecular weight of the polymer, while the volume of the polymer solution had a negligible impact. In combination, increasing the polymer molecular weight and polymer solution concentration resulted in improved film deposition and the formation of robust microcapsules.
\end{abstract}


The resultant polymer microcapsules had a thickness of $\sim 5.5 \mathrm{~nm}$ per bilayer, which is in close agreement with conventionally prepared (quiescent (non-flow) adsorption/centrifugation/wash) LbL capsules. The technique reported herein provides a new way to rapidly generate microcapsules approximately eight times quicker than the conventional means, while also amenable to scale up and mass production.

\section{INTRODUCTION}

Polymer microcapsules have attracted increasing interest as potential artificial cells and bioreactors through the encapsulation of functional units in the hollow capsule interior or by incorporating functional units into the shell wall. ${ }^{1-8}$ Non-biological reactions ${ }^{9,10}$ and sensing ${ }^{11,12}$ can also be performed, but the inherent "soft" nature of the polymer films ${ }^{13}$ composing the microcapsules, in comparison to solid impermeable inorganic films, ${ }^{14}$ uniquely allows biological and biologically-inspired reactions to be controlled. This control gives the microcapsules "celllike" properties ${ }^{8,15}$ and allows for the production of biological materials such as genetic material, ${ }^{1}$ proteins, ${ }^{3}$ or biomolecules. ${ }^{5}$ For example, lipids and liposomes can be incorporated into the capsule wall or into the hollow interior of the capsules, allowing for the encapsulation of cargo such as active enzymes. ${ }^{16}$ Alternatively, lipids can be used as the capsule wall, yielding cell-like capsules capable of encapsulating biological material for the transcription and translation of membrane proteins that provide unidirectional pores. ${ }^{4}$ Additional types of biological cargo can be encapsulated during microcapsule preparation, permitting RNA production from the encapsulated DNA. ${ }^{1}$ Using a similar principle of loading during capsule formation, E. coli can be encapsulated, allowing the microcapsules to function as controlled protein microreactors. ${ }^{3}$ 
There are two common routes for the production of microcapsules capable of mimicking cells. One approach is the use of microfluidics for assembly, which often produces capsules larger than the average mammalian cell $(10-120 \mu \mathrm{m}) .{ }^{17}$ The other approach, layer-by-layer (LbL) assembly, is often used for the production of capsules smaller than mammalian cells. ${ }^{18,19}$ Microfluidic capsule production allows for fine control over certain parameters of the microcapsules, such as template composition and loading capacity; however, it requires custom-built apparatus and is generally limited to specific polymer choices. ${ }^{3,20}$ Although the production of microcapsules in microfluidic systems is rapid and can be automated, the yield scales linearly with time because the capsules are produced individually. Efforts to increase yields from microfluidic devices include the use of parallel or bifurcated channels, and higher flow rates. ${ }^{21}$ On the other hand, LbL assembly is a batch process and can be used to prepare larger numbers of capsules, where the number of capsules can often be increased dramatically without affecting processing time. LbL assembly also allows for a multitude of template types to be used and provides a wider array of polymer choices available for capsule formation. However, the conventional (i.e., non-flow adsorption/centrifugation/wash) LbL coating process is generally labor-intensive and time consuming for particle templates. ${ }^{22,23}$ Recent innovations in LbL assembly utilizing immobilized templates, which include electrophoretic and immersive polymer assembly, EPA ${ }^{24}$ and IPA ${ }^{25}$ respectively, have reduced the labor-intensive nature of this coating process, but they still require specialized, although commercially available, equipment. For LbL and microfluidic microcapsule assembly, the coating of large amounts of templates in a relatively short time frame still remains a challenge. Interestingly, combining the two techniques into one capsule production method may significantly reduce the time necessary for LbL assembly, but it also decreases the quantity of capsules produced, as it is not a batch process. ${ }^{26}$ Some attempts have 
been made to combine the use of closed systems, or reactors, with LbL assembly by utilizing rotating arms to avoid particle aggregation. ${ }^{27}$ However, this technique also required the use of multiple filter membranes for each adsorption step, which led to caking of the membranes.

Inspired by standard processing equipment, we herein report the use of liquid fluidized beds for microcapsule production. This approach requires fewer steps and considerably less time than conventional LbL assembly (Figure 1). An upward flow of water was used to fluidize dispersed particles with the gravitational force being balanced by the drag force of the water. This set-up not only allows for the particles to be suspended and dispersed in a fluidized bed, but also allows for polymers to be passed through the fluidized bed in the upward flow stream (Figure 1). This simultaneously eliminates the need for centrifugation during the washing steps applied in conventional (i.e., non-flow adsorption/centrifugation/wash) LbL assembly and the non-batch production standard to microfluidics. The use of a fluidized bed could be thought of as a “microfluidic” LbL assembly technique. 


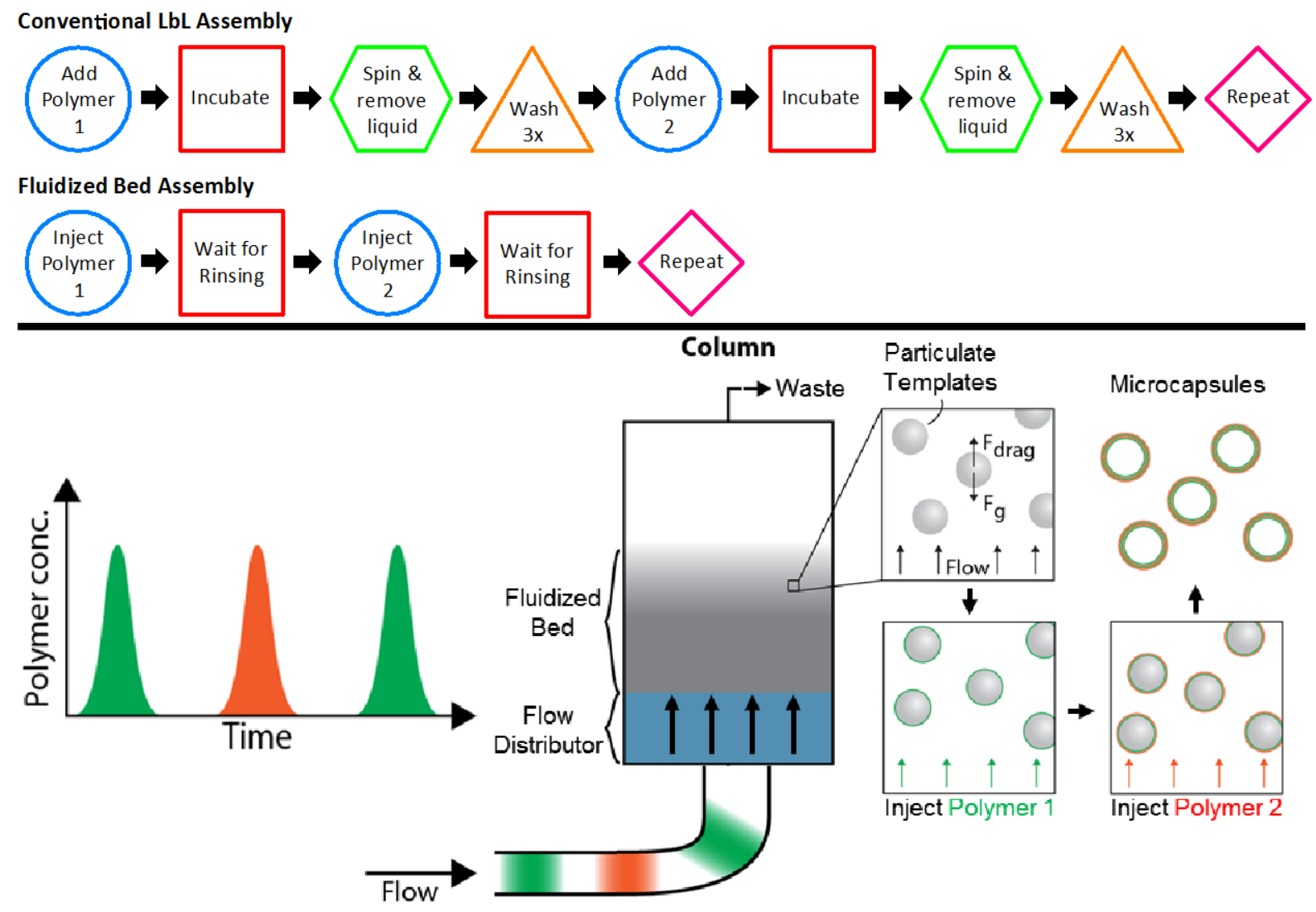

Figure 1. Top: Comparison of the steps required for conventional LbL assembly versus fluidized bed assembly to deposit a polymer bilayer. Bottom: Illustration of the flow stream, fluidized bed and microcapsule formation. The fluidized bed is formed in the column when the force of gravity on the template particles is balanced by the drag force of the flow. Polymer solution can be injected into the flow and is distributed upon entering the column so that the template particles can be uniformly layered. After a sufficiently thick film is generated, the template particles can be removed to yield microcapsules.

\section{MATERIALS AND METHODS}

Fluidization of Template Particles. See Figure S1 for a photograph of the experimental setup. Ultra-pure water with a resistivity greater than $18 \mathrm{M} \Omega \cdot \mathrm{cm}$, from an inline Millipore 
RiOs/Origin purification system, was used. $500 \mathrm{mg}$ of particles $(30-60 \mu \mathrm{m}$ glass beads (predominantly silica, with an average diameter of $50 \mu \mathrm{m}$ ) from Polysciences, Inc., were added into the fluid bed column ( $1 \mathrm{~cm}$ in diameter NAP-5 column from GE Healthsciences) and the pump (PHD2000 from Harvard Apparatus) flow rate was set to $250 \mu \mathrm{L} \mathrm{min}{ }^{-1}$. The height of the fluid bed column was recorded using a caliper and this was repeated with flow rates increasing at $250 \mu \mathrm{L} \mathrm{min}{ }^{-1}$ increments until the particles reached the top of the column, which was $21.35 \mathrm{~mm}$ in height.

Fluidized Bed LbL Capsule Assembly (Reference Conditions). The fluidization of $10 \mathrm{mg}$ of template particles was achieved using a flow rate of $1000 \mu \mathrm{L} \mathrm{min}^{-1}$ of ultra-pure water. A 6-port valve (IDEX Health and Science) was used for injecting the polymers ( 15 000 Da poly(sodium 4-styrenesulfonate) (PSS) from Fluka, and 15 000 Da poly(allylamine hydrochloride) (PAH) from Sigma-Aldrich) into the fluidized bed without disrupting the flow rate of water. A $115 \mathrm{~cm}$, $1.02 \mathrm{~mm}(0.04$ ”) inner diameter polyether ether ketone tube ( 1 $\mathrm{mL}$ volume) was used for loading the polymer solutions. PAH and PSS were prepared in $0.5 \mathrm{M} \mathrm{NaCl}$ (Sigma-Aldrich) pH 7, with a polymer concentration of $0.1 \mathrm{mg} \mathrm{mL}^{-1}$.

The polymer solution was injected into the loading tube using a syringe with the 6-way valve set to "load," isolating the polymer solution from the flow of water. $2 \mathrm{~mL}$ of polymer solution was loaded through the $1 \mathrm{~mL}$ loading tube to ensure that the solution was not diluted. The valve was then set to "inject", diverting the flowing water through the polymer loading tube. The loaded polymer solution was then part of the flow stream and flowed through the fluidized bed. An additional $3 \mathrm{~mL}$ of ultra-pure water flowed through the loading tube and fluidized bed to ensure that both were sufficiently washed before the valve was reset to "load" for the second polymer and the process repeated until eight layers were deposited. Therefore, one 
deposition/wash step took 4 min (1 min for the deposition and 3 min for the wash) and the entire process took $32 \mathrm{~min}$.

Modified Reaction Parameters (High Molecular Weight $\left(\mathbf{M}_{\mathrm{W}}\right)$, High Volume, High Concentration, and Combined High Concentration and High $\left.\mathbf{M}_{\mathbf{W}}\right)$. The experimental conditions were the same as the standard conditions mentioned above except for the differences specifically mentioned below. High $M_{W}$ : High $\mathrm{M}_{\mathrm{W}}$ PSS ( 70 $\left.000 \mathrm{Da}\right)$ and PAH ( 58 $\left.000 \mathrm{Da}\right)$ (both from Sigma-Aldrich) were used instead of the 15000 Da polymers. High Volume: The residence time of the polymer was increased two-fold by injecting polymer solution twice for each layer. High Concentration: The polymer concentration was increased by using $1 \mathrm{mg} \mathrm{mL}^{-1}$ polymer solution instead of $0.1 \mathrm{mg} \mathrm{mL}^{-1}$. Combined High Concentration and High $M_{W}$ : A combination of both high concentration $\left(1.0 \mathrm{mg} \mathrm{mL}^{-1}\right)$ and high $\mathrm{M}_{\mathrm{W}}$ polymers ( 70 000 Da PSS and 58 000 Da PAH) was used. These concentration and polymer $\mathrm{M}_{\mathrm{W}}$ are low enough to keep the solution behaving like a Newtonian Fluid. ${ }^{28}$

Core Removal. The layered particles were sedimented and the supernatant was removed. 300 $\mu \mathrm{L}$ of hydrofluoric acid (5 M HF) was added to the layered particles, the solution was pelleted, the supernatant was removed, and another $300 \mu \mathrm{L}$ of HF was added. [Caution! HF is highly toxic. Extreme care should be taken when handling HF solution.] The HF was washed out by three centrifuge/resuspend/rinse cycles using ultra-pure water. To dissolve the inorganic shell resulting from the use of the glass bead templates, the inorganic/organic composites were further washed twice with $0.5 \mathrm{M}$ hydrochloric acid $(\mathrm{HCl})$. The $\mathrm{HCl}$ was then washed out by three centrifuge/resuspend/rinse cycles using ultra-pure water. 


\section{RESULTS AND DISCUSSION}

Fluidization Calculations. The fluid velocity boundaries in which fluidization can occur for a given particle size lies between the minimum fluidizing velocity $\left(\mathrm{U}_{\mathrm{MF}}\right)$ and the single particle termination velocity or Stokes velocity $\left(\mathrm{U}_{\mathrm{T}}\right)$. $\mathrm{U}_{\mathrm{MF}}$ is the minimum velocity at which a packed or settled bed can be fluidized. Conversely, $\mathrm{U}_{\mathrm{T}}$ is the maximum velocity of a particle in a fluidized bed, above which the fluidized bed will be lost as the individual particle will be carried out of the column by the flow (Figure 2). Thus, the range between $U_{M F}$ and $U_{T}$ can be thought of as the window in which fluidization is possible.

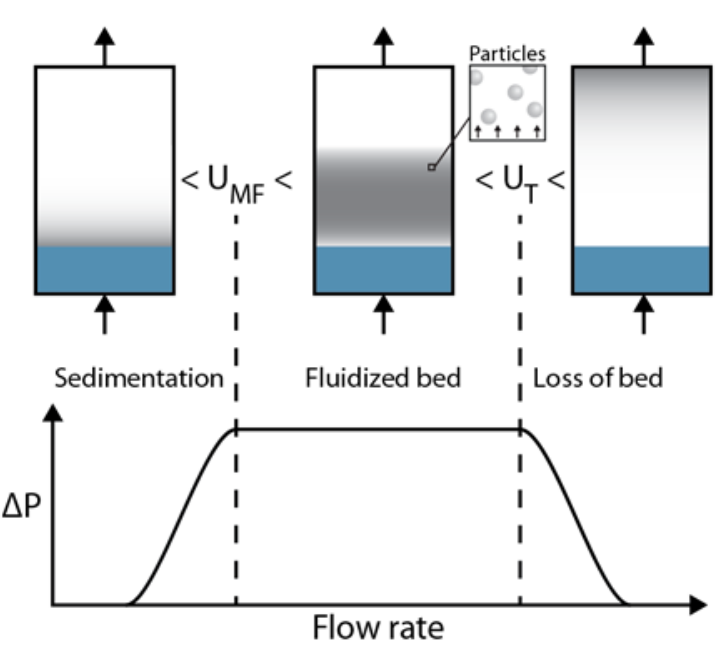

Figure 2. Illustration of the bed at different flow rates. The pressure change experienced when the flow rate passes $U_{\mathrm{MF}}$ corresponds to a release of pressure as the sedimented bed becomes fluidized. Another pressure change is experienced as $U_{T}$ is passed and the drag becomes stronger than gravity, carrying the bed out of the column. Between $U_{M F}$ and $U_{T}$ the pressure does not change as the bed is fully fluidized and in equilibrium.

$\mathrm{U}_{\mathrm{MF}}$ and $\mathrm{U}_{\mathrm{T}}$ can be calculated as follows: ${ }^{29} \mathrm{U}_{\mathrm{T}}$ can be determined using Stokes' law for a system with a low particle Reynolds number $\left(\operatorname{Re}_{\mathrm{p}}\right)$ less than 1.0: 
$U_{T}=\frac{g d_{p}^{2}\left(\rho_{p}-\rho_{f}\right)}{18 \mu}$

where $\mathrm{Re}_{\mathrm{p}}$ is calculated as follows:

$\operatorname{Re}_{p}=\frac{\rho_{f} U_{s} d_{p}}{\mu}$

$\mathrm{U}_{\mathrm{MF}}$ can be found using the following equations:

$$
\begin{aligned}
& A r=150 \frac{(1-\varepsilon)}{\varepsilon^{3}} \operatorname{Re}_{M F}+1.75 \frac{1}{\varepsilon^{2}} \operatorname{Re}_{M F}^{2} \\
& A r=\frac{g d_{p}^{3} \rho_{f}\left(\rho_{p}-\rho_{f}\right)}{\mu^{2}} \\
& U_{M F}=\frac{\mu \operatorname{Re}_{M F}}{\rho_{f} d_{p}}
\end{aligned}
$$

where $A r$ is the Archimedes numbers, $\rho_{f}$ is the density of the fluid (for water, $1000 \mathrm{~kg} \mathrm{~m}^{-3}$ ), $\rho_{p}$ is the density of the particles (for glass beads, $2500 \mathrm{~kg} \mathrm{~m}^{-3}$ ), $\mu$ is the dynamic viscosity of the fluid (for water, $\left.8.9 \times 10^{-4} \mathrm{~Pa} \cdot \mathrm{s}\right), g$ is the force of gravity $\left(9.81 \mathrm{~m} \mathrm{~s}^{-2}\right.$ ), Re is the Reynolds number, $\mathrm{Re}_{p}$ is the Reynolds number of the particles, $\mathrm{Re}_{M F}$ is the Reynolds number at the minimum fluidization velocity, $U_{s}$ is the superficial velocity $\left(\mathrm{m} \mathrm{s}^{-1}\right), \varepsilon$ is the voidage or volume fraction in the fluidized bed, which is not occupied by the particles. Expressed mathematically, voidage $=$ (total volume - volume of the particles) / (total volume), (the calculated value of 0.72 was used), and $d_{p}$ is the average diameter of the particles.

Calculations (Table S1) show that $50 \mu \mathrm{m}$ particles (Figure S2) theoretically fit into the fluidization set-up well, as a large flow rate equates to a high volume of polymer being moved through the system in a short period of time. As shown below, this allows for faster layering. 5 $\mu \mathrm{m}$ particles were theoretically on the border of what was deemed feasible, as any flow rate 
below $100 \mu \mathrm{L} \min ^{-1}$ was considered to be too slow to offer a significant advantage over conventional LbL assembly (Figure 3). Therefore, we needed to determine if the calculated $U_{M F}$ and $\mathrm{U}_{\mathrm{T}}$ values were experimentally valid.

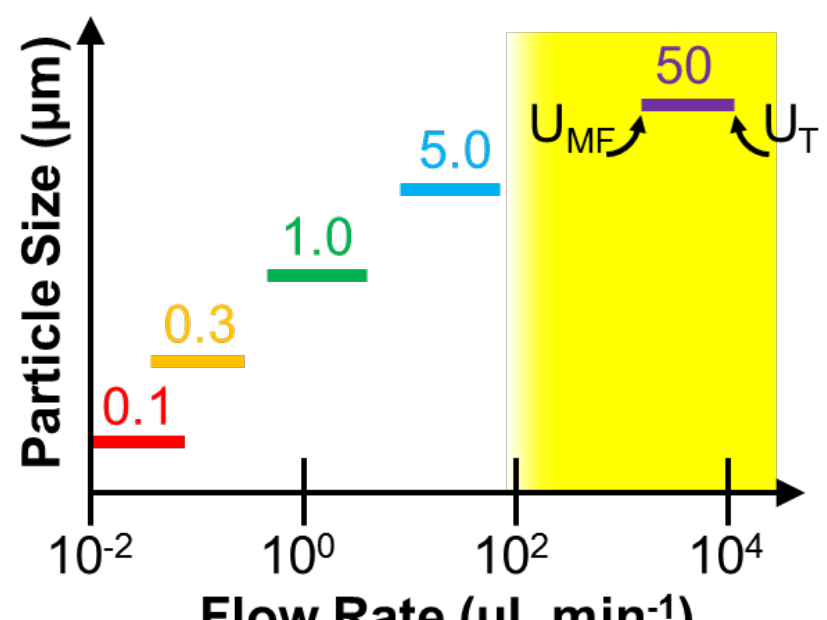

Figure 3. Theoretical fluidization ranges for glass particles of different diameters (in $\mu \mathrm{m}$ ). The yellow range above a flow rate of $10^{2} \mu \mathrm{L} \mathrm{min}{ }^{-1}$ corresponds to the flow rate range necessary for fluidized bed assembly to be advantageous over conventional LbL assembly. See Table S1 for values.

Experimental Determination of Bed Height and Flow Rates. Initial experiments showed that a flow rate of $100 \mu \mathrm{L} \mathrm{min}{ }^{-1}$ was too fast for $5 \mu \mathrm{m}$ particles and did not result in a stable bed, as the templates were carried out of the column by the flow over a period of roughly $30 \mathrm{~min}$. The $5 \mu \mathrm{m}$ silica particles had a lower density, $1800-2000 \mathrm{~kg} \mathrm{~m}^{-3}$, than the $50 \mu \mathrm{m}$ glass particles and therefore would have a theoretical maximum flow rate of $\sim 60-70 \mu \mathrm{L} \min ^{-1}$, suggesting that $\mathrm{U}_{\mathrm{T}}$ is an accurate upper bound for stable bed formation. Therefore, to keep the layering time below that of conventional layering technique, and on the time scale of many microfluidic systems, we 
restricted our analysis to $50 \mu \mathrm{m}$ particles (Figure S2). The experimental flow rates necessary for fluidizing the particles fit in accurately with the calculated values, with the void fraction being controllable between 0.82 and 0.98 , for $250 \mu \mathrm{L} \mathrm{min}^{-1}$ and $4000 \mu \mathrm{L} \mathrm{min}^{-1}$, respectively (Figure 4). Polymer bridging between the particles would not be expected, as there would be roughly 9-fold more free volume than the volume of particles; that is, roughly one diameter length of free space surrounding each particle. ${ }^{29}$ The fluid bed height increased proportionally to the increase in flow rate, and so an intermediary flow rate of $1000 \mu \mathrm{L} \min ^{-1}$ was chosen for further studies. Theoretically, this allows for roughly 1 min of polymer contact per layer and allows for a much faster layering process than conventional (i.e., non-flow adsorption/centrifugation/wash) LbL assembly.

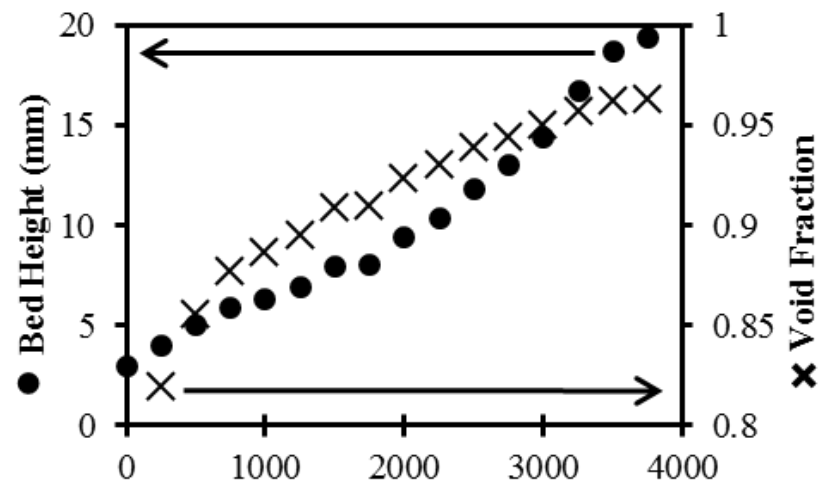

Flow Rate $\left(\mu \mathrm{L} \mathbf{m i n}^{-1}\right)$

Figure 4. Fluidization height and void fraction, determined experimentally using $500 \mathrm{mg}$ of the $50 \mu \mathrm{m}$ particles, plotted against the flow rate of water.

Layer Deposition Using Fluidized Beds. Layering was first performed with reference layering conditions where the polymer is known to be in high excess when compared to the 10 mg of particles in the bed. ${ }^{18}$ Zeta $(\zeta)$-potential analysis in water of the layered particles showed 
that the mean $\zeta$-potential did not alternate between negative and positive with the addition of each layer, as would be expected for LbL assembly with oppositely charged polyelectrolytes (Figure 5 and Figure S3). Literature shows that layering PAH and PSS on silica particles yields $\zeta$-potential values that alternate from $+40 \mathrm{mV}$ to $-40 \mathrm{mV}$, respectively. ${ }^{30}$ This discrepancy with the literature may suggest that the particles were not layered uniformly. Images of the coated particles prior to core removal show that no aggregates were formed during the fluidized bed assembly (Figure S4).

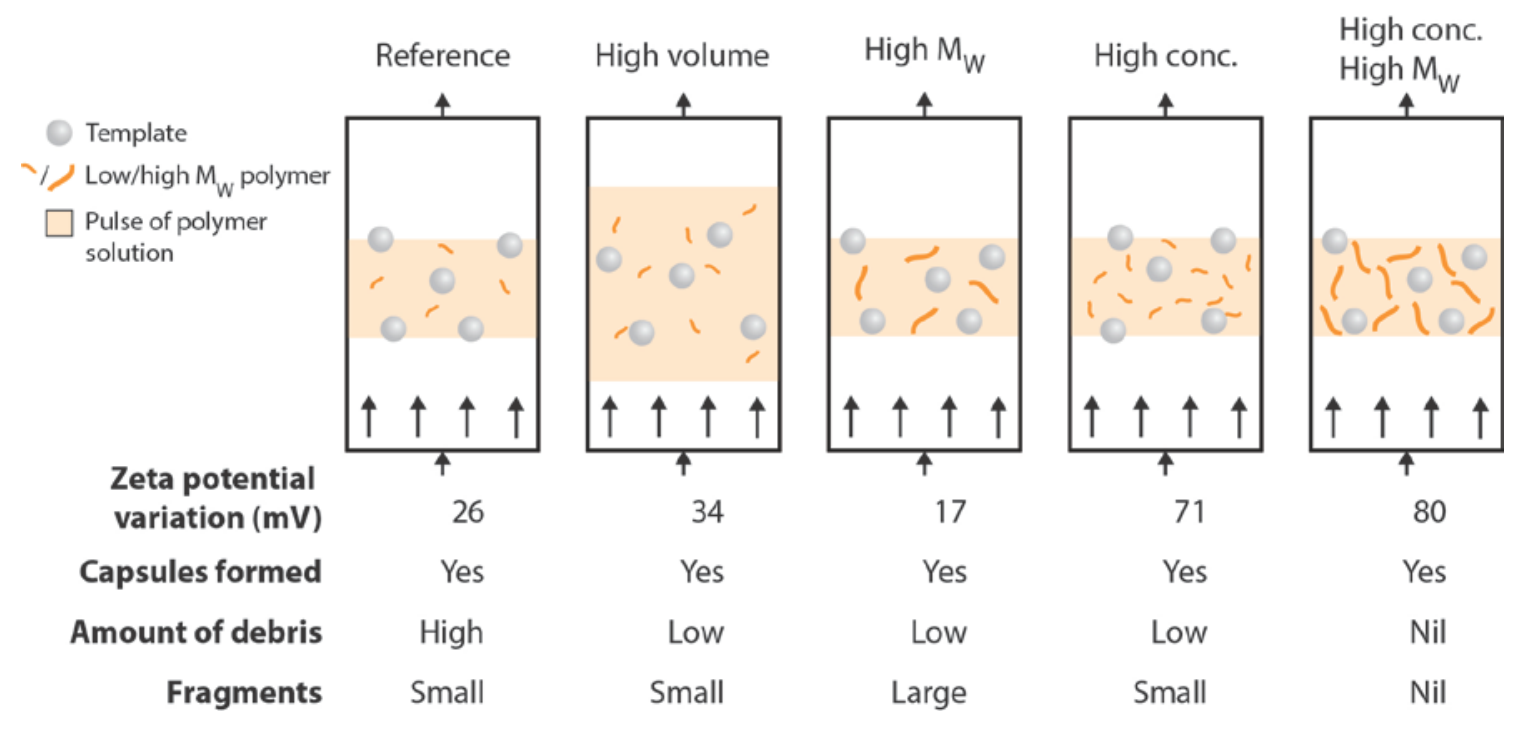

Figure 5. Illustration of the different conditions used for layering and the corresponding experimental results relevant to layer deposition and capsule formation.

To improve layering, the reference conditions were modified by independently varying the $\mathrm{M}_{\mathrm{W}}$ of the polymers, and the volume and concentration of the polymer solutions (Figure 5 and Figure S3). Layering was improved by increasing the $\mathrm{M}_{\mathrm{W}}$ of the polymers. Previous reports show that increasing polymer $\mathrm{M}_{\mathrm{W}}$ leads to thicker films on planar substrates because the long polymer chains can form large loops away from the surface. ${ }^{31}$ Additionally, it has been shown that the 
length (i.e., the $\mathrm{M}_{\mathrm{W}}$ ) of the polymers can affect layering, as shorter polymers have a tendency to be stripped off a surface, particularly for polymers below around $10000 \mathrm{Da},{ }^{31,32}$ which suggests that longer polymers may layer better than shorter polymers. Even though the polymer concentrations are at saturation levels, increasing the concentrations by a factor of 10 resulted in improved layering, as suggested by $\zeta$-potential measurements. Although a concentration of 0.1 $\mathrm{mg} \mathrm{mL} \mathrm{L}^{-1}$ is in excess of the total adsorbable amount, previous reports have also shown that increasing the polymer concentration can increase the layer thickness and the adsorbed amount. $^{33,34}$ Finally, to determine if the layering could be further improved, both the concentration of the polymer solutions and the $\mathrm{M}_{\mathrm{W}}$ of the polymers were increased at the same time. By increasing both the concentration and the $\mathrm{M}_{\mathrm{W}}$ in tandem, improved layering was achieved in the fluidized bed set-up. The mean $\zeta$-potential values were constantly above or below $+30 \mathrm{mV}$ or $-30 \mathrm{mV}$, respectively, similar to the literature values. ${ }^{30}$ This also demonstrates that the charge of the $50 \mu \mathrm{m}$ uncoated glass particles $(-28 \mathrm{mV})$ did not significantly impede layering when compared to the smaller, more highly charged silica $(-40 \mathrm{mV})$ reported in the literature. $^{30}$ The thickness of eight-layer polymer film on the core-shell particles was approximately $20 \mathrm{~nm}$, as determined by transmission electron microscopy (TEM) (Figure S5), and it could be seen from scanning electron microscopy (SEM) (Figure S6) that some of the larger features on the template particles were coated, leading to a smoother surface overall. Energy dispersive X-ray spectroscopy (EDX) was used to confirm that nitrogen (from PAH) and sulfur (from PSS) were both present on the particles (Figure S7).

Capsule Formation Using Fluidized Beds. After HF treatment, we found that polymer capsules were not formed. This could be due to the presence of calcium in the $50 \mu \mathrm{m}$ glass bead 
templates, leading to the formation of inorganic/organic composites. The inorganic/organic composites did not collapse upon drying and had a significantly rougher surface, as visualized by TEM and SEM (Figures S5, S6 and S8). SEM images of the inorganic/organic composites showed small sub-micron-sized lumps (Figure S6), which suggested that the polymer films act as nucleation sites for inorganic particles. EDX was used to map the elemental composition of the inorganic/organic composites and it was found that calcium and fluorine were main components of the inorganic/organic composites, but that nitrogen and sulfur were also present (Figure S9), suggesting that the inorganic/organic composites are composed of a thin organic film of fused inorganic particles. Using charged polymer shells for the nucleation of inorganic materials has been reported in the literature, ${ }^{35}$ including for PAH/PSS microcapsules. ${ }^{36}$ The fact that the inorganic/organic composites did not collapse, ${ }^{36}$ and had a thickness of roughly $500 \mathrm{~nm}$ (Figure S8), suggested that the HF used for template removal crystallized the low amount of calcium ( 5-10\%) present in the template particles and produced inorganic/organic composites.

Hydrochloric acid was used to dissolve the inorganic material from the capsule wall, which greatly reduced the roughness of the capsule surface under differential interference contrast (DIC) microscopy (Figure S10). Additionally, the apparent thickness of the capsules was reduced under DIC (Figure 6). Upon drying, the capsules collapse and display folds representative of conventionally prepared polyelectrolyte capsules. ${ }^{36}$ Atomic force microscopy (AFM) was used to determine the average thickness of five capsules, giving an average minimum shell thickness of $21.8 \pm 4.6 \mathrm{~nm}$, which equates to $2.7 \mathrm{~nm}$ per layer. This value is similar to the thickness estimated from TEM (Figure S5) and is nearly twice that of conventionally prepared PAH/PSS capsules reported in the literature, ${ }^{37}$ but still fits into the range of PAH/PSS capsules prepared using newer techniques $^{24}$ and the thicknesses expected with LbL capsules in general. ${ }^{38}$ These data suggest 
that the $\mathrm{HCl}$ treatment dissolves the inorganic material, leaving the LbL polyelectrolyte shell behind. The root mean square roughness was $9-11 \mathrm{~nm}$, which is rougher than the $\sim 4 \mathrm{~nm}$ usually expected for four bilayer LbL PAH/PSS films; ${ }^{39}$ however, the increased roughness is likely due to the roughness of the original template particles, and the increased thickness of the layers themselves (Figure S6). We note that using pure silica templates, instead of glass templates, could remove the need for the above-described acid treatment.
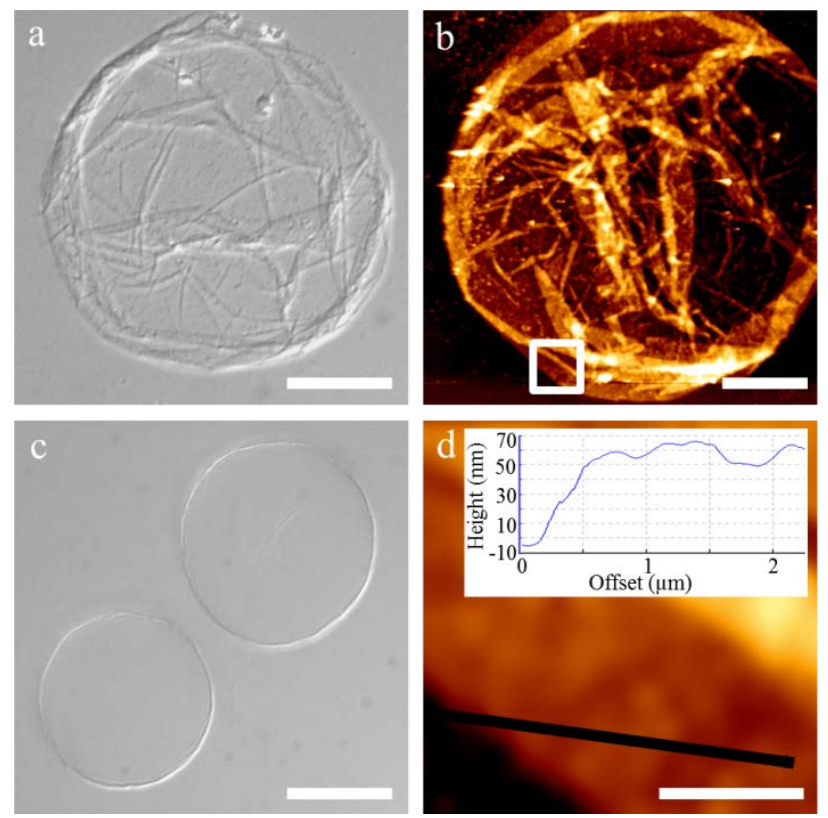

Figure 6. Dry and hydrated four bilayer PAH/PSS capsules prepared via acid treatment after layering in a fluidized bed with the optimized conditions. DIC images of (a) a dry capsule, and (c) hydrated capsules. AFM image of (b) a dry capsule, and (d) magnified region from the box in b along with the height profile across the black line. The scale bars are (a) $15 \mu \mathrm{m}$, (b) $10 \mu \mathrm{m}$, (c) $25 \mu \mathrm{m}$, and (d) $1 \mu \mathrm{m}$.

Using these optimized conditions, $500 \mathrm{mg}$ of glass bead templates were used for a single batch of layering and microcapsules were successfully formed after subsequent core removal and acid 
treatment (Figure 7a). Over 99\% of the particles could be recovered from the column, and 98\% of the original number of particles yielded capsules as counted by optical microscopy. The fluidized bed assembly provides an easy way of rapidly depositing layers of polymer on particulate templates and is roughly eight times quicker than conventional (i.e., non-flow adsorption/centrifugation/wash) LbL assembly (4 min per layer and wash compared to 30 min per layer and wash). ${ }^{18,19}$ Further, successful coating of $500 \mathrm{mg}$ of template in this short time frame indicates the potential for large-scale applicability of this technique.

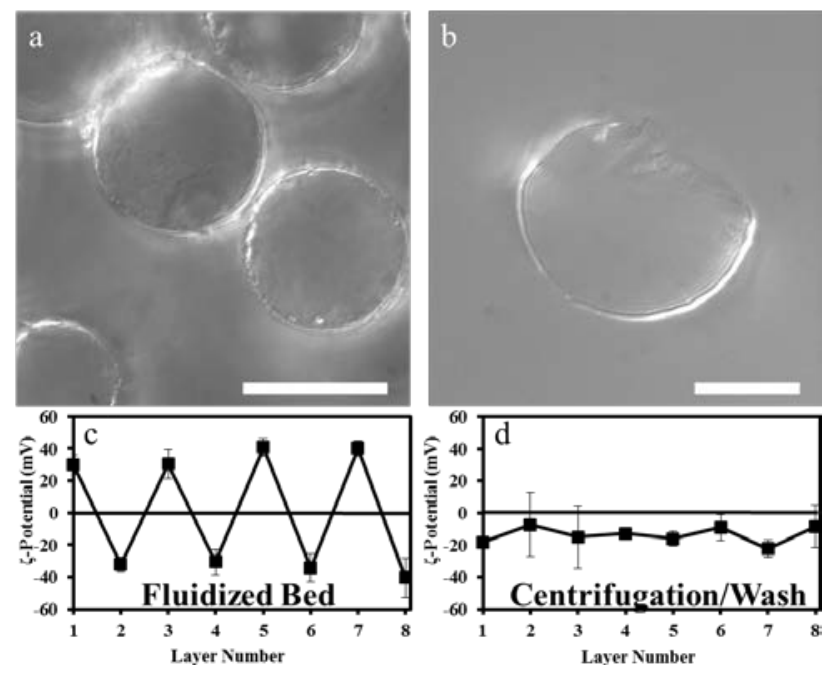

Figure 7. Comparison between capsules prepared by fluidized bed assembly and conventional LbL using a polymer adsorption time of $1 \mathrm{~min}$. DIC images of acid-treated four bilayer PAH/PSS capsules prepared via (a) fluidized bed assembly and (b) conventional LbL assembly. Corresponding $\zeta$-potentials for the core-shell particles prepared via (c) fluidized bed assembly, and (d) conventional LbL assembly. The scale bars are (a) $50 \mu \mathrm{m}$ and (b) $25 \mu \mathrm{m}$.

Conventional LbL assembly. For a comparison and positive control to the fluidized bed layering, conventional (i.e., non-flow adsorption/centrifugation/wash) LbL assembly was used 
for coating the $50 \mu \mathrm{m}$ particles using the optimized fluidized bed conditions. The particles layered with conventional LbL assembly for 1 min per layer did not have alternating positive and negative mean $\zeta$-potentials and only had a $\zeta$-potential difference of $22 \mathrm{mV}$ between the maximum and minimum values, which was significantly narrower than the comparable fluidized bed results (Figure 7). This suggests that 1 min of incubation time under these conditions was not sufficient for optimal polymer deposition, which is supported by evidence that after core removal and acid treatment the resultant capsules were fractured and broken (Figure 7b). Additionally, planar film studies in the literature that used PAH with different polymer combinations, showed that more than 6 min per layer was required for build-up under conventional layering conditions. ${ }^{40}$ Therefore, it is likely that the directional flow of polymer in the fluidized beds allows for improved deposition versus the random deposition in conventional LbL assembly. Other LbL assembly systems have shown rapid layering when directional movement of the particles or templates has been used. ${ }^{26}$ Together with literature data, our results demonstrate that the directional flow of the fluidized bed set up has a strong bearing on the uniformity and density of polymer layering. Fluidization was possible for the $50 \mu \mathrm{m}$ particles, while the timescale necessary for the fluidization of smaller particles $(\leq 5 \mu \mathrm{m}$ in diameter $)$ using the present technique did not provide significant advantages over conventional LbL assembly. To improve upon the conditions for smaller templates, fluidization could be performed in lower density liquids or even gas, which has been performed for silica particles smaller than $10 \mathrm{~nm} .{ }^{41}$ However, the fluidization of nanoparticles in gas often requires the use of acoustic, ${ }^{42}$ magnetic, ${ }^{43}$ or physical disturbances ${ }^{44}$ to break up the large agglomerates that form. ${ }^{45}$ Similarly, using denser templates such as gold would allow for higher $\mathrm{U}_{\mathrm{MF}}$ and $\mathrm{U}_{\mathrm{T}}$ values, making it accessible to particles around $1.5 \mu \mathrm{m}$ (for gold particles with a density of $19300 \mathrm{~kg} \mathrm{~m}^{3}$ ). However, gold 
particles can be difficult to produce as large monodisperse spherical templates and are generally limited to sub $300 \mathrm{~nm}^{46}$

\section{CONCLUSION}

We have introduced fluidized bed column for the efficient LbL assembly of polymers and for the production of microcapsules. This is the first study of particle fluidization in water to form fluidized beds for the deposition of polymer multilayers and the subsequent production of hollow microcapsules. It was found that the experimental flow rate necessary for fluidizing silica matched well with the model calculation and fell between $U_{M F}$ and $U_{T}$. The influence of individual variables such as polymer solution volume, polymer $\mathrm{M}_{\mathrm{W}}$, and concentration was investigated for $50 \mu \mathrm{m}$-diameter particles. The most important variable for polymer film deposition and capsule formation was the concentration of the polymer solution; however, increasing the polymer $\mathrm{M}_{\mathrm{W}}$ along with the polymer solution concentration resulted in uniform film deposition and the formation of robust microcapsules. Core removal with HF resulted in the formation of a thick inorganic/organic composite shell that prevented collapsing upon drying, but could be removed with $\mathrm{HCl}$ to yield stable polymer capsules. Interestingly, it was found that the directed flow in the fluidized beds greatly improved polymer deposition and capsules quality when comparing the fluidized bed set up to conventional (i.e., non-flow adsorption/centrifugation/wash) LbL assembly. This study confirms that directed polymer deposition such as flow in a fluidized bed, similar to an electric current through agarose, ${ }^{24}$ has benefits and complements conventional LbL assembly and microcapsule production. The directed flow in fluidized beds allows for a significant reduction in the processing time required to make LbL microcapsules, from the standard 10-20 min incubation per layer, to $1 \mathrm{~min}$ incubation per layer. This technique opens up the efficient mass-production of large 
microcapsules and further improvements could be accomplished by using automated loading and injection systems.

\section{ASSOCIATED CONTENT}

Supporting Information. Supporting information containing raw values for the calculations along with additional characterization. This material is available free of charge via the Internet at http://pubs.acs.org.

\section{AUTHOR INFORMATION}

Corresponding Author

*E-mail: fcaruso@unimelb.edu.au

Author Contributions

The manuscript was written through contributions of all authors. All authors have given approval to the final version of the manuscript.

\section{ACKNOWLEDGEMENTS}

This work was supported by the Australian Research Council under the Australian Laureate Fellowship (F.C., FL120100030) and the Super Science Fellowship (F.C., FS110200025) schemes, as well as the Australian Government through an Australian Postgraduate Award (M.B.) and an International Postgraduate Research Scholarship (M.B.). 


\section{REFERENCES}

1. Price, A. D.; Zelikin, A. N.; Wark, K. L.; Caruso, F. A Biomolecular "Ship-in-a-Bottle": Continuous RNA Synthesis Within Hollow Polymer Hydrogel Assemblies. Adv. Mater. 2010, 22, 720-723.

2. Peyratout, C. S.; Dähne, L. Tailor-Made Polyelectrolyte Microcapsules: From Multilayers to Smart Containers. Angew. Chem., Int. Ed. 2004, 43, 3762-3783.

3. Zhang, J.; Coulston, R. J.; Jones, S. T.; Geng, J.; Scherman, O. A.; Abell, C. One-step Fabrication of Supramolecular Microcapsules from Microfluidic Droplets. Science 2012, 335, 690-694.

4. Matosevic, S.; Paegel, B. M. Layer-by-Layer Cell Membrane Assembly. Nat. Chem. 2013. 5, 858-963.

5. Qi, W.; Duan, L.; Wang, K.; Yan, X.; Cui, Y.; He, Q.; Li, J. Motor Protein CF0F1 Reconstituted in Lipid-Coated Hemoglobin Microcapsules for ATP Synthesis. Adv. Mater. 2008, 20, 601-605.

6. Trau, D.; Yang, W.; Seydack, M.; Caruso, F.; Yu, N.-T.; Renneberg, R. Nanoencapsulated Microcrystalline Particles for Superamplified Biochemical Assays. Anal. Chem. 2002, 74, 5480-5486.

7. Städler, B.; Price, A. D.; Chandrawati, R.; Hosta-Rigau, L.; Zelikin, A. N.; Caruso, F. Polymer Hydrogel Capsules: en route Toward Synthetic Cellular Systems. Nanoscale 2009, 1, 68-73. 
8. Chang, T. M. S. Therapeutic Applications of Polymeric Artificial Cells. Nat. Rev. Drug Discov. 2005, 4, 221-235.

9. Skirtach, A. G.; De Geest, B. G.; Mamedov, A.; Antipov, A. A.; Kotov, N. A.; Sukhorukov, G. B. Ultrasound Stimulated Release and Catalysis Using Polyelectrolyte Multilayer Capsules. J. Mater. Chem. 2007, 17, 1050-1054.

10. Betancor, L.; Luckarift, H. R. Bioinspired Enzyme Encapsulation for Biocatalysis. Trends Biotechnol. 2008, 26, 10, 566-572.

11. del Mercato, L. L.; Ferraro, M. M.; Baldassarre, F.; Mancarella, S.; Greco, V.; Rinaldi, R.; Leporatti, S. Biological Applications of LbL Multilayer Capsules: From Drug Delivery to Sensing. Adv. Colloid Interface Sci. 2014, DOI: 10.1016/j.cis.2014.02.014

12. Guo, J.; Ping, Y.; Ejima, H.; Alt, K.; Meissner, M.; Richardson, J. J.; Yan, Y.; Peter, K.; Elverfeldt, D. v.; Hagemeyer, C. E. Engineering Multifunctional Capsules through the Assembly of Metal-Phenolic Networks. Angew. Chem. Int. Ed. 2014, 126, 5652-5657.

13. Best, J. P.; Javed, S.; Richardson, J. J.; Cho, K. L.; Kamphuis, M. M.; Caruso, F. Stiffness-Mediated Adhesion of Cervical Cancer Cells to Soft Hydrogel Films. Soft Matter 2013, 9, 4580-4584.

14. Long, Y.; Song, K.; York, D.; Zhang, Z.; Preece, J. A. Engineering the Mechanical and Physical Properties of Organic-Inorganic Composite Microcapsules. Colloid. Surface A 2013, 433, 30-36.

15. Li, J.; Möhwald, H.; An, Z.; Lu, G. Molecular Assembly of Biomimetic Microcapsules. Soft Matter 2005, 1, 259-264. 
16. Chandrawati, R.; Odermatt, P. D.; Chong, S.-F.; Price, A. D.; Städler, B.; Caruso, F. Triggered Cargo Release by Encapsulated Enzymatic Catalysis in Capsosomes. Nano Lett. 2011, 11, 4958-4963.

17. Campbell, N. A.; Williamson, B.; Heyden, R. J. Biology: Exploring Life. Boston, Massachusetts: Pearson Prentice Hall; ISBN 0-13-250882-62006.

18. Caruso, F.; Caruso, R. A.; Möhwald, H. Nanoengineering of Inorganic and Hybrid Hollow Spheres by Colloidal Templating. Science 1998, 282, 1111-1114.

19. Donath, E.; Sukhorukov, G. B.; Caruso, F.; Davis, S. A.; Möhwald, H. Novel Hollow Polymer Shells by Colloid-Templated Assembly of Polyelectrolytes. Angew. Chem. Int. Ed. 1998, 37, 2201-2205.

20. Zhang, H.; Liu, D.; Shahbazi, M. A.; Mäkilä, E.; Herranz-Blanco, B.; Salonen, J.; Hirvonen, J.; Santos, H. A. Fabrication of a Multifunctional Nano-in-micro Drug Delivery Platform by Microfluidic Templated Encapsulation of Porous Silicon in Polymer Matrix. Adv. Mater. 2014, DOI: 10.1002/adma.201400953

21. Teh, S.-Y.; Lin, R.; Hung, L.-H.; Lee, A. P. Droplet Microfluidics. Lab Chip 2008, 8, 198-220.

22. Qi, A.; Chan, P.; Ho, J.; Rajapaksa, A.; Friend, J.; Yeo, L. Template-Free Synthesis and Encapsulation Technique for Layer-by-Layer Polymer Nanocarrier Fabrication. ACS Nano 2011, 5, 9583-9591.

23. Yan, Y.; Björnmalm, M.; Caruso, F. Assembly of Layer-by-Layer Particles and Their Interactions with Biological Systems. Chem. Mater. 2013, 26, 452-460. 
24. Richardson, J. J.; Ejima, H.; Lörcher, S. L.; Liang, K.; Senn, P.; Cui, J.; Caruso, F. Preparation of Nano- and Microcapsules by Electrophoretic Polymer Assembly. Angew. Chem. Int. Ed. 2013, 52, 6455-6458.

25. Richardson, J. J.; Liang, K.; Kempe, K.; Ejima, H.; Cui, J.; Caruso, F. Immersive Polymer Assembly on Immobilized Particles for Automated Capsule Preparation. Adv. Mater. 2013, 25, 6874-6878.

26. Kantak, C.; Beyer, S.; Yobas, L.; Bansal, T.; Trau, D. A 'Microfluidic Pinball' for OnChip Generation of Layer-by-Layer Polyelectrolyte Microcapsules. Lab Chip 2011, 11, 10301035.

27. Voigt, A.; Lichtenfeld, H.; Sukhorukov, G. B.; Zastrow, H.; Donath, E.; Baumler, H.; Möhwald, H. Membrane filtration for microencapsulation and microcapsules fabrication by Layer-by-Layer polyelectrolyte adsorption. Ind. Eng. Chem. Res. 1999, 38, 4037-4043.

28. Harrison, G.; Franks, G. V.; Tirtaatmadja, V.; Boger, D. V. Suspensions and Polymers Common Links in Rheology. Korea-Aust. Rheol. J. 1999, 11. 197-218.

29. Richardson, J. F.; Harker, J. H.; Backhurst, J. R. Coulson and Richardson's chemical engineering: Particle technology and separation processes. Chemical Engineering Vol. 2. Butterworth-Heinemann, 2002.

30. Smith, R. N.; McCormick, M.; Barrett, C. J.; Reven, L.; Spiess, H. W. NMR Studies of PAH/PSS Polyelectrolyte Multilayers Adsorbed onto Silica. Macromolecules 2004, 37, 48304838. 
31. Sui, Z.; Salloum, D.; Schlenoff, J. B. Effect of Molecular Weight on the Construction of Polelctrolyte Multilayers: Stripping versus Sticking. Langmuir 2003, 19, 2491-2495.

32. Sui, Z.; Schlenoff, J. B. Molecular Weight Effects on the Construction of Polyelectrolyte Multilayers. Abstr. Pap. Am. Chem. S. 2001, 221, U414-U414.

33. Cheung, J.; Stockton, W.; Rubner, M. Molecular-Level Processing of Conjugated Polymers. 3. Layer-by-Layer Manipulation of Polyaniline via Electrostatic Interactions. Macromolecules 1997, 30, 2712-2716.

34. Parfitt, G. D.; Rochester, C. H. Adsorption from Solution at the Solid/Liquid Interface. Academic Press London 1983.

35. Butler, M. F.; Frith, W. J.; Rawlins, C.; Weaver, A. C.; Heppenstall-Butler, M. Hollow Calcium Carbonate Microsphere Formation in the Presence of Biopolymers and Additives. Cryst. Growth Des. 2008, 9, 534-545.

36. Shchukin, D. G.; Sukhorukov, G. B.; Möhwald, H. Smart Inorganic/Organic Nanocomposite Hollow Microcapsules. Angew. Chem. Int. Ed. 2003, 42, 4472-4475.

37. Leporatti, S.; Voigt, A.; Mitlohner, R.; Sukhorukov, G.; Donath, E.; Möhwald, H. Scanning Force Microscopy Investigation of Polyelectrolyte Nano- and Microcapsule Wall Texture. Langmuir 2000, 16, 4059-4063.

38. De Geest, B. G.; Déjugnat, C.; Prevot, M.; Sukhorukov, G. B.; Demeester, J.; De Smedt, S. C. Self-Rupturing and Hollow Microcapsules Prepared from Bio-polyelectrolyte-Coated Microgels. Adv. Funct. Mater. 2007, 17, 531-537. 
39. Lowman, G. M.; Buratto, S. K. Nanoscale Morphology of Polyelectrolyte SelfAssembled Films Probed by Scanning Force and Near-Field Scanning Optical Microscopy. Thin Solid Films 2002, 405, 135-140.

40. Lvov, Y.; Decher, G.; Möhwald, H. Assembly, Structural Characterization, and Thermal Behavior of Layer-by-Layer Deposited Ultrathin Films of Poly(Vinyl Sulfate) and Poly(Allylamine). Langmuir 1993, 9, 481-486.

41. Liu, H.; Guo, Q.; Chen, S. Sound-Assisted Fluidization of $\mathrm{SiO}_{2}$ Nanoparticles with Different Surface Properties. Ind. Eng. Chem. Res. 2007, 46, 1345-1349.

42. Ammendola, P.; Chirone, R. Aeration and Mixing Behaviours of Nano-Sized Powders Under Sound Vibration. Powder Technol. 2010, 201, 49-56.

43. Zeng, P.; Zhou, T.; Yang, J. Behavior of Mixtures of Nano-Particles in Magnetically Assisted Fluidized Bed. Chem. Eng. and Proces. 2008, 47, 101-108.

44. Yang, J.; Zhou, T.; Song, L. Agglomerating Vibro-Fluidization Behavior of NanoParticles. Adv. Powder Tech. 2009, 20, 158-163.

45. van Ommen, J. R.; Valverde, J. M.; Pfeffer, R. Fluidization of Nanopowders: A Review. J. Nanopart. Res. 2012, 14, 1-29.

46. Bastuaccents, N. G.; Comenge, J.; Puntes, V. Kinetically Controlled Seeded Growth Synthesis of Citrate-Stabilized Gold Nanoparticles of up to $200 \mathrm{~nm}$ : Size Focusing versus Ostwald Ripening. Langmuir 2011, 27, 11098-11105. 
TOC

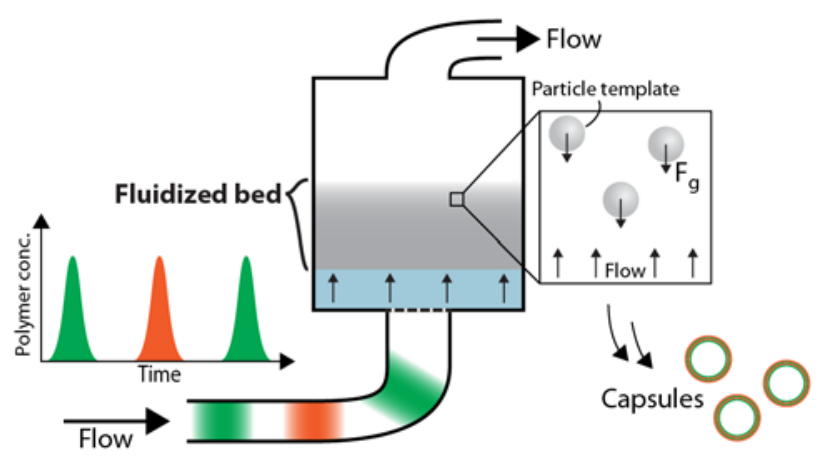




\section{University Library}

\section{- M M N E R VA A gateway to Melbourne's research publications}

Minerva Access is the Institutional Repository of The University of Melbourne

Author/s:

Richardson, JJ;Teng, D;Bjoernmalm, M;Gunawan, ST;Guo, J;Cui, J;Franks, GV;Caruso, F

Title:

Fluidized Bed Layer-by-Layer Microcapsule Formation

Date:

2014-08-26

Citation:

Richardson, J. J., Teng, D., Bjoernmalm, M., Gunawan, S. T., Guo, J., Cui, J., Franks, G. V. \& Caruso, F. (2014). Fluidized Bed Layer-by-Layer Microcapsule Formation. LANGMUIR, 30 (33), pp.10028-10034. https://doi.org/10.1021/la502176g.

Persistent Link:

http://hdl.handle.net/11343/111986 